\title{
Aprender geometría a partir del ordenamiento de ideas
}

\author{
Franklin Edmundo Molina Jiménez \\ Universidad Central del Ecuador \\ femolina@uce.edu.ec
}

Recibido: 9 -febrero-2018/ Aceptado: 31 - julio-2018

\section{Resumen}

Este trabajo tiene como objetivo analizar dos modelos didácticos que contribuyen al proceso de enseñanza aprendizaje de la geometría, además conocer el marco histórico de evolución de la geometría y sus aplicaciones en otras ramas del saber humano. Se fundamenta en una investigación bibliográfica descriptiva y explicativa, información que permitió conocer el proceso evolutivo de la geometría en paralelo al de la humanidad y su importancia para el desarrollo de las matemáticas, se presenta un ejemplo de aplicación propio del autor, en el que se presentan los niveles de aprendizaje de la geometría a través del modelo de Van Hiele, permitiendo establecer que el aprender geometría implica desarrollar nuevas estructuras mentales a partir de ordenar ideas ya existentes, que se convertirán en transcendentales, cuando el estudiante logra relacionarlas con otras ciencias desarrolladas por el hombre.

Palabras clave: modelos didácticos para geometría, evolución de la geometría, estructuras mentales.

\section{Abstract}

The objective of this work is to analyze two didactic models that contribute to the process of teaching geometry learning, as well as to know the historical framework of evolution of the geometry and its applications in other branches 
of the human knowledge. It is based on a descriptive and explanatory bibliographic research, information that allowed to know the process of evolution of the geometry in parallel to that of the humanity and its importance for the development of the mathematics, there appears a proper example of application of the author, in which there are detailed the levels of learning of the geometry across the model of Van Hiele, allowing to establish that learning geometry implies developing new mental structures from arranging already existing ideas, which will become transcendental, when the student manages to relate them with other sciences developed by the man.

Keywords: didactic models for geometry, evolution of the geometry, mental structures. 


\section{Introducción}

prender geometría, a partir del ordenamiento de ideas, busca profundizar en
el estudio de metodologías relacionadas con el aprendizaje de la geometría,
comprendiendo cómo las ideas generadas en nuestro cerebro se van ordenando en procesos mentales, que son observables y verificables, en el momento que los seres humanos pueden resolver diversos problemas de diferentes temáticas y específicamente los relacionados con las formas geométricas creadas de forma natural o artificial por los hombres, que rodean al mundo que habitamos.

Se debe iniciar entendiendo que la geometría según Báez e Iglesias, (2007) ha sido considerada como uno de los pilares de formación académica y cultural del hombre, dada su aplicación en diversos contextos y su capacidad formadora del razonamiento lógico, razón fundamental para profundizar en analizar los procesos metodológicos para su enseñanza.

Mientras que las ideas surgen de dos fuentes según Locke (1698): de las experiencias que son producto de las sensaciones y de las relaciones con las observaciones que hacemos sobre los objetos sensibles externos y las percepciones que son procesos de la mente, de sus propias operaciones y de los modos de ellas y debido a las cuales llega el entendimiento a tener ideas sobre dichas operaciones. Confirmando que una idea no es más que una imagen que existe o se forma en la mente, capacidad humana relacionadas con la autorreflexión, la creatividad y la habilidad de adquirir y aplicar el intelecto, además las ideas dan lugar a la formación de conceptos, los cuales son la base de cualquier tipo de conocimiento, tanto científico como filosófico.

En el desarrollo del presente documento, se profundizará en el análisis de cómo estos dos conceptos se relacionan entre ellos. Es necesario conocer que las reformas curriculares dadas en el Ecuador en los últimos años, han considerado desarrollar las destrezas con criterio de desempeño en el aprendizaje en diversas áreas del conocimiento, siendo una de ellas la geometría, sin embargo a pesar de esto, los maestros relegan los contenidos de geometría al final del curso, ocasionando que algunos temas sean revisados de manera superficial o no sean tratados, ya que priorizan la enseñanza de la matemática (Abrate, Delgado y Pochulu, 2006).

Según datos del estudio, Aportes para la enseńanza de la Matemática, realizado por la Unesco en América Latina en el año 2016 (Unesco, 2016), el 43\% de los estudiantes de sexto grado y el $51 \%$ de los estudiantes de tercer grado de la educación general básica media del Ecuador lograron resolver los ítems relacionados al dominio geometría en la prueba TERCE (Tercer Estudio Regional Comparativo y Explicativo).

En el Ecuador la institución que busca promover una educación de excelencia evaluando integralmente al Sistema Nacional de Educación, es el Sistema Nacional de Evaluación (Ineval), quien al entregar los resultados de la evaluación Ser Bachiller ciclo 2016-2017 determinó que los niveles de logros alcanzados por los 
estudiantes a nivel nacional, en el dominio matemática en el tópico relacionado a los temas aplicación de progresiones aritméticas y geométricas, el porcentaje de aciertos en los ítems, corresponde a menos del 50\% y en el tópico relacionado a los temas de resolución de problemas con perímetros y áreas apenas supera al 50\% (Ineval, 2017).

Datos que nos permiten confirmar que el proceso de enseñanza aprendizaje tradicional de la geometría se concentra en aprender de memoria las fórmulas para el cálculo de áreas de figuras geométricas, volúmenes de cuerpos geométricos, definiciones, propiedades y teoremas geométricos, apuntalados con la utilización de gráficos construidos en forma mecánica y en ocasiones sin una estructura lógica, ocasionando que los jóvenes, cuando llegan a la universidad deban enfrentarse a situaciones que no siempre están al alcance de su comprensión.

La información obtenida se puede verificar de fuentes de datos bibliográficos tales como artículos académicos, científicos y libros con rigor científico que admiten ser cotejados, además se han considerado criterios que permiten la inclusión y exclusión de temas pertinentes a la geometría y su didáctica.

En la presente investigación se tiene el propósito de identificar y describir algunos modelos que desarrollan el proceso de enseñanza aprendizaje a través de aplicaciones didácticas del medio, reflexionando sobre la importancia de aprender geometría aplicando uno de estos modelos didácticos.

\section{La geometría en el transcurso del tiempo}

La geometría a lo largo del tiempo ha ido perfeccionándose, los esquemas mentales de los hombres dedicados al estudio de esta rama de la matemática, se han estructurado en esquemas cada vez más elaborados y complejos, Piaget (1978) señala que un esquema, expresa el conjunto organizado de características que permiten repetir las acciones del sujeto o aplicarlas a contenidos nuevos; un esquema es una totalidad fuerte e integrada, tal que los comportamientos que induce están íntimamente relacionados. La formación del conocimiento geométrico en el plano como en el espacio está relacionada con los periodos históricos en los que ha ido evolucionando los esquemas mentales, por lo que es necesario presentar una breve reseña histórica para comprender su evolución.

Las mediciones de los objetos son tomadas como las primeras nociones de la geometría, siendo estas empíricas, los egipcios y babilonios utilizando la estrategia del tanteo dedujeron fórmulas del cálculo de perímetros y áreas. Los procesos de razonamiento deductivo fueron impuestos por los griegos, ya que realizaron conclusiones a través de demostraciones lógicas y procesos experimentales. Eudoxo, Euclides, Arquímedes, Apolonio y Pappus, presentaron las primeras nociones geométricas que con el pasar del tiempo se convirtieron en los cimientos de la geometría.

La inscripción y la circunscripción de figuras rectas poligonales en figuras curvas permitió establecer el concepto de límite analizado por Eudoxo (408-355 a.C.), 
Euclides (300 a.C.) estableció los procesos lógicos de la geometría al establecer un razonamiento deductivo en sus demostraciones, desarrollando axiomas y postulados que relacionan al punto y al segmento de recta, legado, utilizado hasta la presente por desarrollar el razonamiento hipotético-deductivo fundamentado en el método axiomático, conocida como la geometría euclidiana, inmortalizada en su obra magistral Elementos.

El procedimiento geométrico de llegar a un resultado con un grado de precisión que aumenta a medida que su cálculo avanza, es considerado en el método exhaustivo empleado por Arquímedes (287-212 a.C.) en sus demostraciones. La idea de coordenada permitió que Apolonio (262-200 a.C.) establezca los primeros estudios de la geometría analítica, fundamentados en las demostraciones sobre propiedades de las cónicas. La geometría proyectiva la estableció Pappus (300 d.C.) argumentando la relación anarmónica o la correspondencia que se establece en forma lineal entre el álgebra y la geometría.

Durante los siglos XVII y XVIII, Descartes (1591-1661) se encargó de desarrollar la geometría analítica, en la que se realiza el análisis de las figuras geométricas a partir de un sistema de coordenadas relacionando métodos algebraicos y análisis matemáticos, Fermat (1601-1665) considero que una ecuación con dos incógnitas determina una curva plana.

En el siglo XIX, Plucker (1801-1868) estudió los espacios n-dimensionales relacionándolos con el álgebra, definiendo las coordenadas trilineales.

En el siglo XVII, la geometría proyectiva se hace presente con mayor rigurosidad cuando Descartes y Pascal (1623-1662) representaron bidimensionalmente un objeto tridimensional, a través de utilizar propiedades proyectivas. Desargues (15911661) utilizó la relación armónica al demostrar su teorema de los dos triángulos.

La geometría descriptiva la construyó Monge (1746-1818) utilizando una técnica gráfica, fundamentada en la proyección ortogonal, en la que un sólido tridimensional se determina a partir de sus proyecciones en tres planos al dibujar sus vistas laterales.

La geometría no euclidiana es fundamentada por Saccheri (1667-1733), Lambert (1728-1777) y Legendre (1752-1833), quienes construyeron modelos geométricos, para demostrar la negación del postulado euclídeano, por un punto exterior a una recta dada, puede trazarse una sola paralela. Gauss (1777-1855), Bolyai (18021860) y Lobachevky (1793-1856), se encargaron de desarrollar la geometría hiperbólica fundamentada en el álgebra lineal.

La jerarquización de la geometría la establece Klein (1849-1925), por medio de subgrupos del grupo de transformaciones de geometrías afines, presentadas en su obra, El programa de Erlangen.

Esta descripción histórica de la geometría muestra la manera cómo ha ido evolucionando, de una manera dinámica soportada por la interacción entre procesos de visualización (ligados al pensamiento espacial), procesos de justificación (ligados 
al pensamiento deductivo) y aplicaciones instrumentales que se llevan a cabo con el objeto de resolver problemas de la vida cotidiana, de las ciencias o de la misma matemática (Castiblanco et al., 2004, p. 9). Esquemas mentales que sirven de base y de columna vertebral de la matemática.

\section{La importancia de aprender geometría}

La geometría es una de las grandes realizaciones de la mente humana, más de 2000 años el hombre ha considerado necesario su estudio, todo ser humano en cualquier tipo de ocupación tiene en alguna ocasión la necesidad de recurrir a la geometría y en algunos campos de estudio esta ciencia es importante en el quehacer profesional, tales como la física, la matemática, la química, la estadística, en la geología, en la economía, la psicología, debido a que es eminentemente práctica.

El hombre refleja en su quehacer diario y en sus obras de arte, esas imágenes ideales que obtiene de la observación de la naturaleza: realiza objetos de cerámica, dibujos, edificios y lo más diversos utensilios proyectando en ellos las figuras geométricas que ha perfeccionado en la mente, así lo afirma Godino (2005). Gracias al estudio de la geometría se puede tener el conocimiento necesario para poder penetrar profundamente en los detalles y complejidades maravillosos de nuestro universo.

Vivimos en un mundo llamado la era del conocimiento y de la información, a pesar de que una persona no estudie una carrera científica, es necesario que tenga algún conocimiento científico y el estudio de la geometría permite tener alguna comprensión básica del mundo que nos rodea.

Para Piaget (1978), las primeras interacciones del niño pequeño con su entorno, previas al desarrollo del lenguaje, se basan casi totalmente en experiencias espaciales, muy en particular a través de los sentidos de la vista y el tacto. Afirmando que al ir desarrollando en los estudiantes su intelecto la geometría le permite razonar lógicamente, argumentar sus afirmaciones y demostrarlas, además de comprobar las proposiciones por razonamiento deductivo o inductivo, analizando un problema en términos de los datos que se ven, las leyes y principios que pueden aceptarse como verdaderos mediante una reflexión cuidadosa, lógica y exacta, para seleccionar una solución del problema.

\section{Modelos didácticos que permiten aprender geometría}

Aunque parte de la importancia de la enseńanza de la geometría radica en ser la disciplina donde los estudiantes llevan a cabo procesos de razonamiento, pareciera que la realidad en las aulas es distinta, pues uno de los problemas en la enseñanza de la geometría es la dificultad que existe para que los estudiantes pasen de la descripción de las figuras a un proceso más formal, basado en razonamientos y argumentación (Castiblanco et al., 2004). Es así que se establece la necesidad de analizar dos modelos didácticos que permitan aprender geometría. 
El primero en considerar es el modelo de la teoría de Van Hiele, que se fundamenta en el trabajo realizado por los esposos Pierre Van Hiele y Dina Van Hielle-Geldof, en Holanda, al ser desarrollado con sus estudiantes, durante el proceso de enseńanza de la geometría.

El modelo de Van Hiele está distribuido en cinco niveles de razonamiento en los que se considera a los conceptos geométricos de diferente manera según el estudiante va avanzando en los diferentes años de estudio de la matemática durante su vida académica.

En el primer nivel llamado, Visualización o reconocimiento, el estudiante reconoce las figuras geométricas a través de su forma como un todo, no identifica partes ni elementos de esta, puede reconocerla y reproducirla. No es capaz de identificar las propiedades de la figura y las compara con elementos conocidos del medio donde habita. No está en la capacidad de nombrar a la figura por su nombre utilizando un lenguaje geométrico.

Para el segundo nivel citado como Análisis, el estudiante ya puede reconocer y analizar los elementos y propiedades de las figuras geométricas, pero no puede definirlas ni establecer clasificaciones entre propiedades con otras figuras similares. A través de la manipulación de objetos, puede establecer propiedades de estas en forma empírica.

En el tercer nivel denominado Ordenación o clasificación, el estudiante ya puede nombrar a las figuras al identificar sus propiedades, establece la relación entre las propiedades que se derivan de otra. Puede encontrar las relaciones entre figuras geométricas similares, dando una definición estructurada a ellas. El razonamiento lógico del estudiante sigue basado en la manipulación del objeto, por lo que no es capaz de organizar una secuencia lógica de razonamientos para realizar una demostración utilizando el sistema axiomático de las matemáticas. Pero sí es capaz de comprender que una propiedad se deriva de otra.

El estudiante en el cuarto nivel llamado Deducción formal, ya realiza deducciones, demostraciones lógicas y formales, como parte del proceso de un razonamiento deductivo. Comprende y relaciona las propiedades, axiomas, teoremas que fundamentan a la geometría. Relaciona proposiciones para deducir propiedades, lo que le permite realizar distintas demostraciones para obtener un mismo resultado.

Finalmente, en el quinto nivel nombrado como Rigor, el estudiante puede analizar el rigor de varios sistemas deductivos para realizar comparaciones entre ellas. Tiene la capacidad de apreciar la consistencia, independencia y complejidad de los axiomas que fundamentan la geometría. Comprende a la geometría en forma abstracta, siendo este nivel desarrollado en las aulas universitarias.

El segundo modelo didáctico analizado, es el desarrollado por Raymond Duval catedrático de la Universidad Louis Pasteur de Estamburgo, en el que divide el aprendizaje de la geometría en tres niveles de razonamiento. 
Duval (1998), citado por Castiblanco et al., 2004, señala que, en el primer nivel denominado, Global de percepción visual, el estudiante puede relacionar figuras con objetos físicos, donde se destaca la forma total de la imagen a partir de la posición o el tipo de trazo.

En el segundo nivel llamado, De percepción de elementos constitutivos, donde el estudiante no solo percibe la imagen globalmente, sino formada por elementos de una misma dimensión o inferiores. En este nivel la posición o tamaño no son importantes pues la atención se centra en establecer las relaciones entre los elementos que conforman la imagen.

En el tercer nivel conocido como Operativo de percepción visual, el estudiante puede operar sobre las figuras, es decir permite la manipulación mental de los elementos constitutivos de ésta.

La propuesta de Duval representa otra base teórica que intenta categorizar el razonamiento de los estudiantes con respecto al aprendizaje de la geometría en las aulas de las instituciones educativas.

Tanto el modelo de Van Hiele como el de Duval, hacen una generalización de las características de las figuras geométricas y de los objetos generando un aprendizaje por medio de percepciones visuales, los conceptos e imágenes son concretados a partir del análisis de sus características, y los estudiantes logran ordenar sus ideas a través de procesos mentales que le permiten realizar justificaciones de propiedades observables utilizando axiomas y teoremas en construcciones geométricas a través del manejo mental de estas, generando y demostrando nuevos conceptos utilizando esquemas mentales ya establecidos.

\section{Aplicación de los niveles de aprendizaje en la geometría}

Se puede afirmar que las estructuras mentales, desarrolladas por los educandos durante los ańos que cursan las aulas, tanto de Bachillerato como las de la Universidad en las que han estructurado nuevos esquemas mentales sobre la base de los conocimientos adquiridos por el aprendizaje de la geometría, sirven de base para la comprensión de nuevos conocimientos relacionados con la matemática, física y otras asignaturas en las que involucre razonamientos matemáticos.

Jones (2002), señala que enseñar geometría implica reconocer y seleccionar problemas geométricos interesantes, teoremas, apreciar el contexto cultural e histórico de la geometría y comprender la variedad de usos y contextos en los cuales está presente la geometría. Afirmación que permite presentar el siguiente ejemplo que es propuesto por el autor, en el cual se evidencia el proceso de formación de las estructuras mentales en función de cada uno de los niveles de razonamiento que propone Van Hiele para el proceso de aprendizaje de la geometría.

En el nivel uno, los estudiantes conocen que dos segmentos son congruentes cuando tienen igual medida sus longitudes, dos ángulos son congruentes cuando tienen igual medida sus aberturas, dos rectas son paralelas cuando dos líneas que 
mantienen una distancia entre ellas, y a pesar de prolongarse, su trayectoria hasta el infinito, nunca llegan a toparse en ningún punto. Si dos rectas paralelas son cortadas por una transversal, los ángulos que se encuentran entre las paralelas a distinto lado de ellas y a distinto lado de la transversal de denominan ángulos alternos internos.

Los estudiantes en el nivel dos reconocen que un cuadrilátero es una figura geométrica formada por cuatro lados. Los distintos cuadriláteros que existen en geometría dependen de la longitud de los lados y de la amplitud de los ángulos que lo forman. La forma básica del cuadrilátero es el cuadrado en el que sus cuatro lados y ángulos internos son congruentes, la segunda figura básica conocida es el rectángulo con dos lados congruentes de dos en dos y sus cuatro ángulos internos congruentes, en estas figuras geométricas sus lados opuestos son paralelos y sus ángulos congruentes miden noventa grados. Estas figuras geométricas pueden ser observadas a nuestro alrededor, en una hoja de papel en la pantalla del televisor o simplemente en una mesa.

En el tercer nivel el estudiante puede comprender que en un cuadrilátero la congruencia entre ángulos opuestos implica el paralelismo de los lados opuestos, también puede afirmar que dos triángulos son congruentes cuando sus tres lados son congruentes cumpliendo con el teorema lado, lado, lado.

En el cuarto nivel de razonamiento, el estudiante demuestra que los lados opuestos $\mathrm{AB}$ y $\mathrm{CD}$ del cuadrilátero $\mathrm{ABCD}$ son paralelos. Para ello debe construir en el cuadrilátero $\mathrm{ABCD}$, un segmento de recta $\mathrm{CB}$, que es una de las diagonales del cuadrilátero, lo que determina que se formen dos triángulos $\triangle \mathrm{CAB}$ y $\triangle \mathrm{BDC}$ que son congruentes por cumplir con el teorema de congruencia lado, lado, lado. Por ser partes correspondientes de dos triángulos congruentes, los ángulos $\mathrm{ABC}$ y $\mathrm{DCB}$ son congruentes. Per-

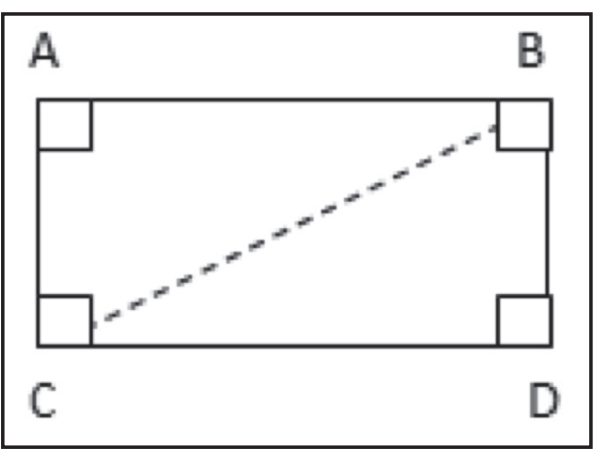
mitiendo llegar a la conclusión que los lados opuestos $\mathrm{AB}$ y $\mathrm{CD}$ son paralelos ya que se considera que los ángulos $\mathrm{ABC}$ y $\mathrm{DCB}$ cumplen con la definición de ser ángulos alternos internos, entre dos rectas paralelas intersecadas por una transversal.

Para el quinto nivel de razonamiento propuesto por Van Hiele, los estudiantes integran sus conocimientos con situaciones del medio, relacionando las definiciones y teoremas geométricos con otras ciencias como por ejemplo en la literatura, en la que Ojeda, (2012) presenta: La historia es circular y la felicidad un triángulo de cuatro lados, nunca podré calcular el diámetro de nuestros abrazos ni la fórmula que resuelve el perímetro de cada despedida.

En la cultura ecuatoriana la geometría, se encuentra plasmada en las prendas de vestir como las polleras, las blusas y las huscarinas que utilizan las mujeres de Suscal, uno de los tres cantones de Cańar, en las que bordan diseños de paisajes, estrellas, patos flores y figuras geométricas de diferentes tamaños y colores. 
En la astronomía, la geometría analítica se encuentra presente al estudiar las órbitas de los planetas alrededor del sol, estas orbitas son secciones cónicas denominadas elipses o hipérbolas. Otras formas geométricas son analizadas cuando se estudia las constelaciones.

\section{Conclusiones}

Para Jones (2002), enseñar geometría implica reconocer y seleccionar problemas geométricos interesantes, teoremas, apreciar el contexto cultural e histórico de la geometría y comprender la variedad de usos y contextos en los cuales está presente la geometría. El docente debe inferir que enseñar geometría implica ir desarrollando nuevas estructuras mentales a partir de ordenar ideas ya existentes, que se convertirán en transcendentales, cuando el estudiante logre relacionarlos con otros contenidos y determine su aplicabilidad en el medio en el cual habita, además el estudiante logra desarrollar un razonamiento lógico y un pensamiento crítico progresivo, conforme el avance del estudio de la geometría se da siempre y cuando el docente este convencido de utilizar un nuevo enfoque para enseñar está rama de la matemática.

Determinar cómo se ha ido modificando el conocimiento de la geometría en el transcurso de los años permite evidenciar la transformación de las estructuras mentales de quienes se dedican al estudio de esta ciencia, que es una rama de la matemática.

Se evidenció que tanto en los modelos de aprendizaje de Van Hiele, como en el de Duval, el enseñar y aprender geometría involucra procesos de razonamientos que van de los más simples a los más complejos, que deben ser desarrollados con la guía del docente, permitiendo considerar que el nivel de aprendizaje de la geometría en los estudiantes estará en función del número de ellos en las aulas de clase de las instituciones educativas, es decir, en aulas con un excesivo número de estudiantes se dificultará que se logre desarrollar estos procesos de la mejor manera.

El docente debe generar ambientes de aprendizaje amigables, en los cuales se determinará la importancia de estudiar geometría, detallar el marco histórico que rodea a la concreción de los conceptos geométricos, consensuar las estrategias de aprendizaje a ser utilizadas para desarrollar el pensamiento lógico matemático y lo más importante establecer la relación y su aplicabilidad con otras ramas del conocimiento como la literatura, el arte, la astronomía, la física, etc.

\section{Referencias}

Abrate, R., Delgado, G. y Pochulu, M. (2006). Caracterización de las actividades de geometría que proponen los textos de matemática. Revista Iberoamericana de Educación, 39(1), 1-9. Recuperado de: http://www.rieoei.org/deloslectores/1290Abrate.pdf 
Almeida, M. (2002). Desarrollo profesional docente en geometría: análisis de un proceso de formación a distancia. Memoria de la tesis doctoral. Departamento de Didáctica de las Ciencias Experimentales y de las Matemáticas. Universidad de Barcelona. Recuperado de: http://www.uv.es/aprengeom/archivos2/Almeida02.pdf

Báez, R. \& Iglesias, M. (2007). Principios didácticos a seguir en el proceso de enseñanza y aprendizaje de la geometría en la UPEL "El Mácaro". Enseñanza de la Matemática. Vols. 12 al 16. Número extraordinario. pp. 67-87.

Castiblanco, A., Urquina, H., Camargo, L. \& Acosta, M. (2004). Pensamiento geométrico y tecnologías computacionales. Colombia: Ministerio de Educación Nacional, Enlace Editores Ltda.

Duval, R. (1998). Geometry from a cognitive point of view. En C. Mammana \& V.Villani (Eds.), Perspective on the Teaching of the Geometry for the 21st Century (pp. 37-51). Dordrecht, Netherlands: Kluwer Academic Publishers.

Godino, J. (2005). Modelo teórico, epistemológico, antropológico y psicológico de la enseñanza de la matemática. Editorial Iberoamérica. Recuperado de: http://www. ugr.es/-jgodino/edumat-maestros/manual/4_Geometria.pdf

Ineval, (2007). Informe de resultados Ser Bachiller ciclo 2016-2017. Recuperado de: www.evaluacion.gob.ec/evaluaciones

Jones, K. (2002). Issues in the Teaching and Learning of Geometry. En L. Haggarty (Ed.), Aspects of Teaching Secondary Mathematics: perspectives on practice. London: Routledge Falmer. Pp.121-139.

Locke, J. (1689). Ensayo sobre el entendimiento humano. Bibliotheque universelle editada por Jean Leclerc. Recuperado de: http://getafe.es/wp-content/uploads/ Locke_John-Ensayo_sobre_el_entendimiento_humano.pdf

Ojeda, D. (2012). Literatura y poesía 2.0. Geometría. Recuperado de: https://literaturaypoesia20.blogspot.com/2012/04/geometria.html

Piaget, Jean. (1978). Introducción a la epistemología genética, 1. El pensamiento matemático. 2a . ed. Buenos Aires: Paidós.

Unesco, Organización de las Naciones Unidas para la Educación, la Ciencia y la Cultura. (2016). Aportes para la enseñanza de la Matemática. Recuperado de: http://unesdoc.unesco.org/images/0024/002448/244855s.pdf. 